\title{
Cerebral Infarctions and Brain Abscess due to Lemierre Syndrome
}

\author{
Yoko ShibAsaki WarabI, Hiroko YoshiKawA*, Jiro IDEZUKA, \\ Motoyoshi YAMAZAKI and Yoji ONISHI
}

\begin{abstract}
Lemierre syndrome is characterized by anaerobic bacterial infection in the head and neck, causing thrombophlebitis of the jugular vein and septic metastasis to various distant organs, most often the lungs. Here we describe previously unreported clinical features of cerebral infarctions and brain abscess due to metastatic septic embolism of this uncommon but lethal syndrome, and the successful course of treatment with ampicillin, cefotaxime and metronidazole.
\end{abstract}

(Internal Medicine 44: 653-656, 2005)

Key words: cerebral infarction, brain abscess, Lemierre syndrome, venous thrombosis, septic embolism, metronidazole

\section{Introduction}

Lemierre syndrome or postanginal septicemia is characterized by anaerobic bacterial infection in the head and neck, causing thrombophlebitis of the jugular vein and septic metastasis to various distant organs, most often the lungs (1). Lemierre syndrome is usually associated with anaerobic infection by Fusobacterium necrophorum, and in the preantibiotic era, this syndrome resulted in the death of previously healthy young people. Today in the post-antibiotic era, observations of this syndrome have been decreasing and the features of central nervous system complications of this syndrome remain uncertain. We report a case of Lemierre syndrome complicating teeth caries, causing thrombophlebitis of the external jugular vein and metastatic cerebral septic embolisms and abscess.

\section{Case Report}

A 59-year-old man presented with headache and fever. $\mathrm{He}$ had been relatively healthy with past medical histories of an appendectomy 30 years earlier, glaucoma 2 years earlier and hyperlipidemia diagnosed 1 year earlier. One week before admission he began to experience chills. The next morning, a prickling temporal pain of the right side developed and by evening, the sensation had changed to a persistent throbbing pain accompanied by low grade fever. Two days before admission dentoalveolitis and right maxillary abscess were diagnosed. Brain MRI was unremarkable. Analgesic administration was not effective. Therefore, he was admitted to our hospital.

On physical examination, his body temperature was 37.0 ${ }^{\circ} \mathrm{C}$, and his throat was slightly inflammed. Three teeth had dental caries and all needed to be extracted. Neurological examination demonstrated only headache. Right temporal pain persisted and expanded to the left side.

On laboratory examination, white blood cell count and Creactive protein were abnormally elevated. Blood chemical test demonstrated liver and renal dysfunction. Blood coagulation and bleeding function were also abnormal (Table 1). Lumbar puncture showed normal findings at that time. Angiography of the brain and neck was performed to rule out temporal arteritis.

Cefazolin (2 $\mathrm{g}$ daily) was given intravenously, but swelling in the left temporal region and the left side of his face and neck developed and gradually increased. Thereafter, the left external jugular vein became a palpable strand. On hospital day 7 , panipenem/ betamipron ( $1 \mathrm{~g}$ daily) was given, but there was no response. CT showed swelling of the left temporal muscle, soft tissue and air passage, with venous thromboses of the left external jugular vein and peritonsillar vein (Fig. 1). Chest CT disclosed multiple small nodular densities throughout both upper lung fields (Fig. 2). Transthoracic echocardiogram was unremarkable.

From the Department of Neurology and *the Department of Infectious disease, Niigata City General Hospital, Niigata

Received for publication August 31, 2004; Accepted for publication January 30, 2005

Reprint requests should be addressed to Dr. Yoko S. Warabi, the Department of Neurology, Tokyo Metropolitan Neurological Hospital, 2-6-1 Musashidai, Fuchu, Tokyo 183-0042 

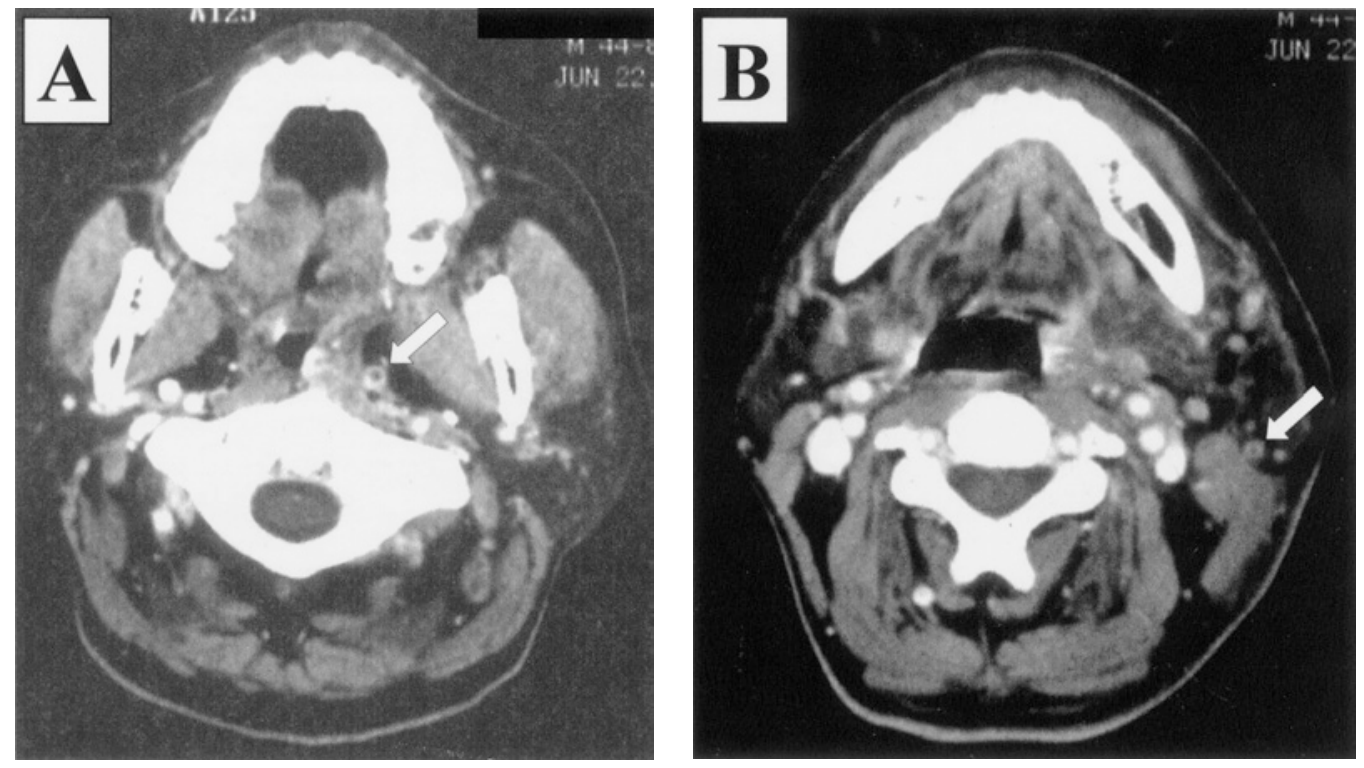

Figure 1. CT showing swelling of the left temporal muscle, soft tissue and air passage and venous thromboses of peritonsillar vein (A) and left external jugular vein (B). Since these veins were obstructed, there was no intravenous contrast medium (arrows).

Table 1. Hematologic and Blood Chemical Data on Admission

\begin{tabular}{llc}
\hline Red blood cells & $\left(\times 10^{4} / \mu \mathrm{l}\right)$ & 486 \\
White blood cells & $(\mathrm{per} \mu \mathrm{l})$ & 13,600 \\
$\quad$ neutrophils & $(\%)$ & 85.2 \\
lymphocytes & $(\%)$ & 6.5 \\
$\quad$ monocytes & $(\%)$ & 7.9 \\
$\quad$ basophils & $(\%)$ & 0.2 \\
$\quad$ eosinophils & $(\%)$ & 0.2 \\
Platelet & $\left(\times 10^{4} / \mu \mathrm{l}\right)$ & 23.3 \\
Fibrinogen & $(\mathrm{mg} / \mathrm{dl})$ & 865 \\
Fibrin degradation product & $(\mu \mathrm{g} / \mathrm{ml})$ & 11.2 \\
& & \\
Blood urea nitrogen & $(\mathrm{mg} / \mathrm{dl})$ & 22.7 \\
Creatinine & $(\mathrm{mg} / \mathrm{dl})$ & 1.1 \\
Aspartate aminotransferase & $(\mathrm{IU} / l)$ & 59 \\
Alanine aminotransferase & $(\mathrm{IU} / \mathrm{l})$ & 103 \\
Alkaline phosphatase & $(\mathrm{IU} / \mathrm{l})$ & 340 \\
Lactate dehydrogenase & $(\mathrm{IU} / \mathrm{l})$ & 381 \\
Cholinesterase & $(\mathrm{IU} / \mathrm{l})$ & 108 \\
& & \\
C-reactive protein & $(\mathrm{mg} / \mathrm{dl})$ & 22.9 \\
Erythrocyte sedimentation rate & $(\mathrm{mm} / \mathrm{h})$ & 62 \\
\hline
\end{tabular}

On hospital day 9, although the patient did not show any signs of fever, neck stiffness or worsening of headache, he was not roused from an afternoon nap. He was noted to have developed consciousness disturbance and right hemiparesis. Low density areas in the left caudate nucleus, the left superior temporal gyrus and the left parietal lobe were detected on brain CT (Fig. 3A). Cerebral infarctions, especially
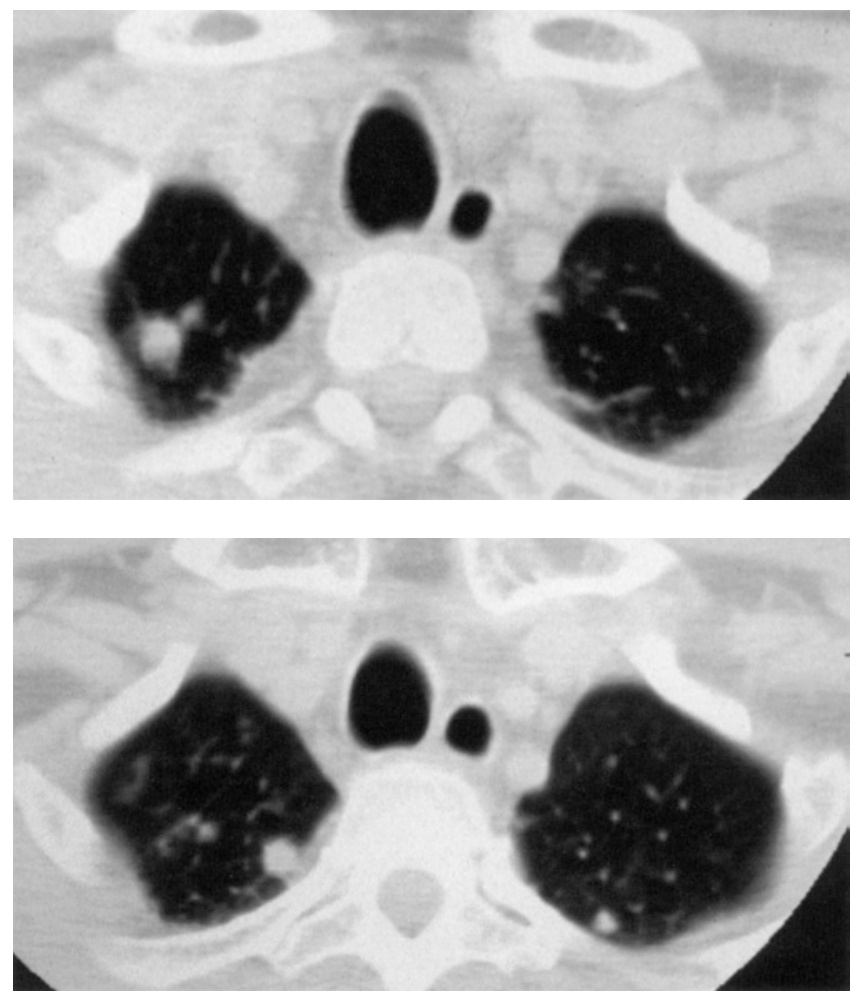

Figure 2. Chest CT disclosing multiple small nodular densities throughout both upper lung fields. Metastatic abcesses in lung were diagnosed. 

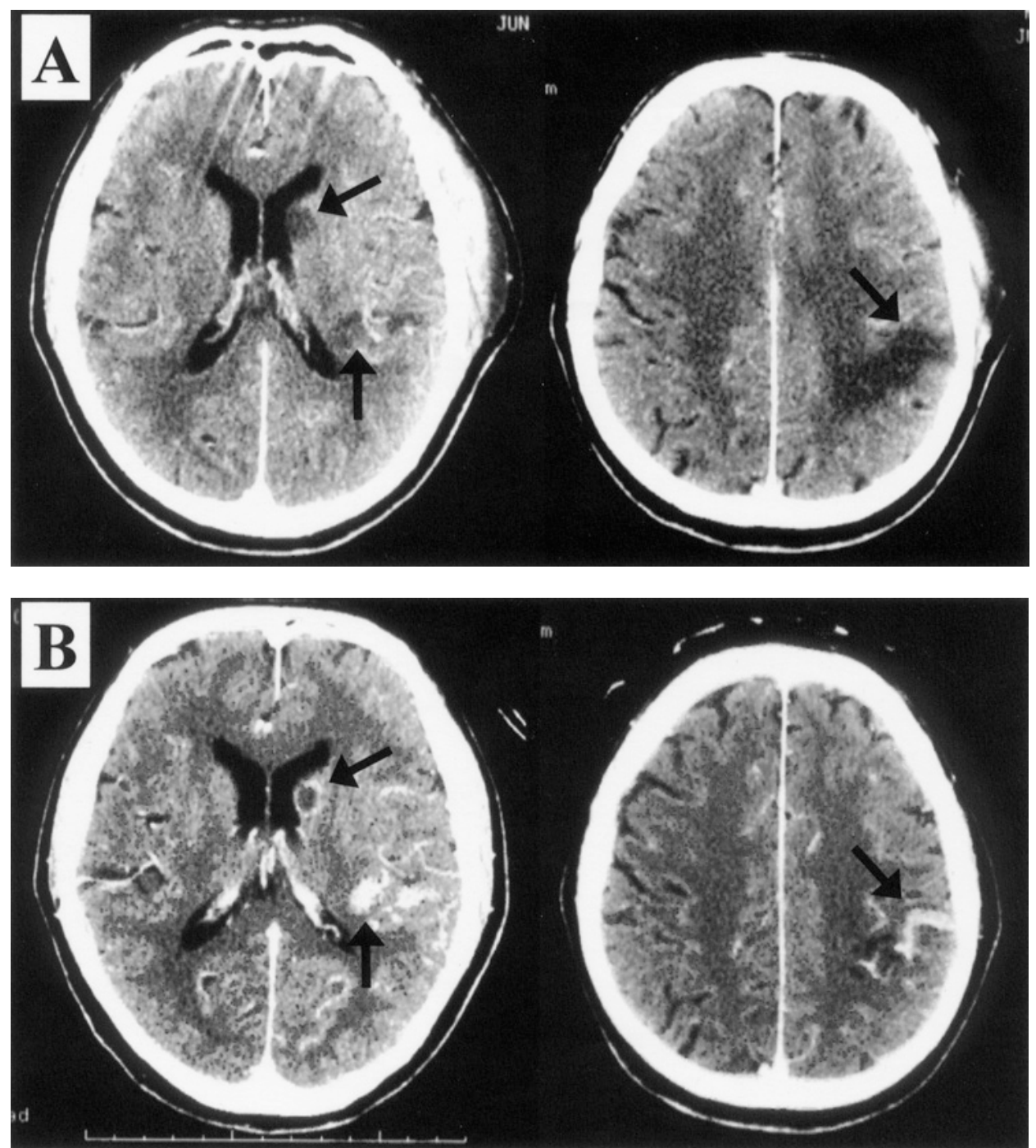

Figure 3. (A) Brain CT showing low density areas in the left caudate nucleus, the left superior temporal gyrus and left parietal lobe on hospital day 9 (arrows). Cerebral infarctions, especially cerebral embolisms were diagnosed. (B) These lesions demonstrated contrast enhancement on hospital day 16 (arrows). Particularly, the left caudate nucleus demonstrated ring-like enhancement which was thought to be a brain abcess. Low density areas of left superior temporal gyrus and parietal lobe appeared to decrease in size and these enhanced areas were thought to be the subacute signs of peripheries of infarcts.

cerebral embolisms were diagnosed. Heparin sodium $(12,000$ unit daily) and clindamycin phosphate (1.2 g daily) were added intravenously. However, on hospital day 16, cerebral lesions became enhanced, and particularly that in the left caudate nucleus demonstrated a ring-like enhancement which was thought to be a brain abcess (Fig. 3B). Left superior temporal gyrus lesion and parietal lobe lesion were also enhanced, but these low density areas appeared to decrease in size and these enhancements were thought to be the subacute signs of peripheries of infarcts. On the same day, lumbar puncture showed an opening pressure of $22 \mathrm{cmH}_{2} \mathrm{O}$, 627 white blood cells (polymorphonuclear cells were 57\%), protein $237 \mathrm{mg} / \mathrm{dl}$, CSF glucose/serum glucose 0.27 , and culture was negative.

Brain abscess of the left caudate nucleus was diagnosed, and then ampicillin ( $8 \mathrm{~g}$ daily), cefotaxime ( $4 \mathrm{~g}$ daily) and metronidazole ( $2 \mathrm{~g}$ daily) were started and given for 7 weeks. Fever, temporal pain and swelling of the head and neck decreased gradually and repeat lumbar puncture showed improvement. During this course, the patient developed facial nerve palsy and hearing disturbance on the right side on hospital day 33. MRI demonstrated thickening of the meninges at the right cerebellopontine angle. Prednisolone was given orally and these findings were improved. 


\section{Discussion}

Lemierre syndrome was first described by Courmont et al in 1900 (2), and then became known as Lemierre syndrome after Lemierre published reviews describing cases of postanginal septicemia $(1,3,4)$. This syndrome is characterized by the following clinical features: it usually affects healthy young adults or adolescents; a tonsillar or peritonsillar abscess acts as the initial cause and spreads to thrombophlebitis of the tonsillar and peritonsillar veins; these thrombophlebitis can spread to that of the internal jugular vein which leads to septicaemia and forms distant metastatic abscesses. The most frequent localizations of such secondary metastatic abscesses are in the lungs. It was reported that $95 \%$ of patients with this syndrome demonstrated pleuro-pulmonary involvement $(5,6)$. Also those abscesses can occur in the large joints, bone, muscle, soft tissues, liver, spleen, kidney, pericardium and endocardium. Meanwhile, the initial causes are not limited to tonsillar or peritonsillar abscess but also include pharyngitis, dental infection, otitis media and mastoiditis, which impair the mucosal defense system.

The present patient was diagnosed with Lemierre syndrome based on the initial dental caries, strong pain and swelling of head and neck, venous thromboses of external jugular vein and peritonsillar vein, and metastatic abscess formation in lung. Furthermore we consider that the cerebral infarctions and subsequent brain abscess were also associated with this course of Lemierre syndrome. The fact that those cerebral infarctions developed at the same time despite different blood supply systems suggests that these were cerebral embolisms. Moreover, it is obvious that these embolisms were septic metastases, because contrast-enhanced brain CT demonstrated ring-like enhancement in one of the same regions as cerebral infarctions and lumbar puncture showed evidence of bacterial infection. Therefore the brain lesions might be associated with Lemierre syndrome.

Little has been described about central nervous system involvement in Lemierre syndrome in previous reports. There are several descriptions of meningitis caused by F. necrophorum particularly in children (8-10). Spencer et al (11) reported a case of $F$. necrophorum sepsis with cerebral infarction in a 23-month-old female infant who did not demonstrate any signs of thrombophlebitis. Stokroos et al (5) described a case of Lemierre syndrome with sigmoid sinus thrombosis, and referred to the possibility of metastatic abscess in the brain or meninges. The present case is important because of the rarity of the condition in that cerebral infarctions and brain abscess were complicated with Lemierre syndrome. However, venous thrombophlebitis usually does not lead directly to arterial infarctions. We suspect that the most likely cause is right-to-left cardiac shunt. Although the trans- thoracic echocardiogram findings in our patient were unremarkable, transesophageal echocardiography had to be examined.

Lemierre syndrome is usually caused by Fusobacterium necrophorum, an anaerobic, nonpathogenic gram-negative rod present in normal mucosal flora. It is sensitive to penicillins, clindamycin, metronidazole, and chloramphenicol (7). Although repeated cultures of blood, sputum and cerebrospinal fluid were negative in our patient, it is possible that the pathogen was an anaerobe such as F. necroforum because it was responsive to penicillin and metronidazole. In the preantibiotic era, this syndrome was associated with a mortality rate of greater than $90 \%$. Today that has been reduced to $4 \%-12 \%$, but the prognosis depends on early diagnosis and adequate treatment, so there is significant morbidity remaining (5).

Lemierre syndrome has been termed "a forgotten disease" in the era of modern antimicrobial therapy. However, it should be considered that dental caries or tonsillitis could progress to this uncommon but lethal syndrome, and furthermore, to cerebral infarction or brain abscess.

\section{References}

1) Lemierre A. On certain septicaemias due to anaerobic organisms. Lancet 1: 701-703, 1936.

2) Courmont P, Cade A. Sur une septico-pyohemie de l'homme simulant la peste et causee par un streptobacille anaerobie. Arch Med Exp Anat Pathol 4: 17-28, 1900.

3) Stallworth JR, Carroll JM. Lemierre's syndrome: New insights into an old disease. Clin Pediatr 36: 715-717, 1997.

4) Sasaki Y, Iwata H, Kinoshita M, Sumiya M. Lemierre syndrome with extensive cervical venous thrombosis and multiple pulmonary embolisms. Nihon Naika Gakkai Zasshi (J Jpn Soc Intern Med) 89: 2174 2176, 2000 (in Japanese).

5) Stokroos RJ, Manni JJ, de Kruijk JR, Soudijn ER. Lemierre syndrome and acute mastoiditis. Arch Otolaryngol Head Neck Surg 125: 589$591,1999$.

6) Moreno S, Garcia Altozano J, Pinilla B, et al. Lemierre's disease: Postanginal bacteremia and pulmonary involvement caused by Fusobacterium necrophorum. Rev Infect Dis 11: 319-324, 1989.

7) Vohra A, Saiz E, Ratzan KR. A young woman with a sore throat, septicaemia, and respiratory failure. Lancet 350: 928, 1997.

8) Bader-Meunier B, Pinto G, Tardieu M, Pariente D, Bobin S, Dommergues J-P. Mastoiditis, meningitis and venous sinus thrombosis caused by Fusobacterium necrophorum. Eur J Pediatr 153: 339-341, 1994.

9) Larsen PD, Chartrand SA, Adickes ED. Fusobacterium necrophorum meningitis associated with cerebral vessel thrombosis. Pediatr Infect Dis J 16: 330-331, 1997.

10) Vogel LC, Boyer KM. Metastatic complications of Fusobacterium necrophorum sepsis. Two cases of Lemierre's postanginal septicemia. Am J Dis Child 134: 356-358, 1980.

11) Spencer CH, Slusher CW, Sanders CV, Aldridge KE. Fusobacterium necrophorum sepsis with cerebral infarction. South Med J 82: 10401043, 1989. 